\title{
BIOLOGÍA Y ECOLOGÍA DECUATRO ESPECIES MEDICINALES DE GENTIANELLA RECOLECTADAS para el mercado en la Región Cajamarca, Perú
}

\section{Biology and ecology of four medicinal species of Gentianella collected for the market in the Cajamarca Region, Peru}

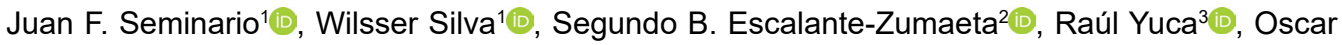 \\ Reátegui ${ }^{4}$ iD \& Ivan Best ${ }^{5}$ (iD
}

\begin{abstract}
Resumen: Se describió la biología y ecología de cuatro especies medicinales de Gentianella (Gentianaceae), con énfasis en aspectos relacionados con la producción, incluyendo el análisis de flavonoides totales. Todas las especies evaluadas fueron hierbas pequeñas. Gentianella graminea y G. crassicaulis son perennes y $G$. dianthoides y $G$. bicolor son anuales. Mientras que $G$. dianthoides produce abundantes semillas, las otras tienen muy escasa a moderada producción de semillas; todas con bajo poder germinativo. Gentianella crassicaulis produce hasta $90 \mathrm{~g}$ de materia seca/ planta, mientras que las restantes menos de $10 \mathrm{~g}$ de materia seca/planta. Gentianella graminea presentó niveles significativamente mayores de flavonoides totales en hoja y tallo comparado con $G$. dianthoides y $G$. crassicaulis $(p<0,05)$. Tres especies (G. graminea, G. crassicaulis y G. dianthoides) habitan en la región Jalca (2700 a 2850 m s.n.m.), asociadas a 30 especies (16 también medicinales); mientras que G. bicolor habita en la región Quechua (2600 a 2750 m s.n.m.), asociada a 17 especies (9 medicinales). Las cuatro especies tuvieron poblaciones escasas ( $\leq$ 2,5 plantas $/ \mathrm{m}^{2}$ ) y su regeneración natural fue baja (1-4 plantas $/ \mathrm{m}^{2}$ ). Gentianella dianthoides y G. crassicaulis se encuentran en estado vulnerable de conservación, por lo que se recomienda suspender su recolección.
\end{abstract}

Palabras clave: Etnobotánica, flavonoides, Gentianaceae, Jalca, Quechua.

Summary: The biology and ecology of four medicinal species of Gentianella (Gentianaceae) were described, with emphasis on aspects related to production, including the analysis of total flavonoids. All the evaluated species were small grasses. Gentianella graminea and G. crassicaulis are perennial and G. dianthoides and G. bicolor are annual. While G. dianthoides produces abundant seeds, the others have little to moderate seed production, all with low germination capacity. Gentianella crassicaulis produces up to $90 \mathrm{~g}$ of dry matter/plant, while the rest produces less than 10 $\mathrm{g}$ of dry matter/plant. Gentianella graminea presented significantly higher levels of total flavonoids in leaves and stems compared to G. dianthoides and G. crassicaulis $(p<0,05)$. Three species (G. graminea, G. crassicaulis and G. dianthoides) occur in the Jalca region (2700 to $2850 \mathrm{~m}$ a.s.I.), along with other 30 species (of which 16 medicinal); while G. bicolor inhabits the Quechua region (2600 a $2750 \mathrm{~m}$ a.s.I.), along with 17 species (9 medicinal). The four species had scarce populations $\left(\leq 2,5\right.$ plants $\left./ \mathrm{m}^{2}\right)$ and their natural regeneration was low (1-4 plants $\left./ \mathrm{m}^{2}\right)$. Gentianella dianthoides and $G$. crassicaulis are in a vulnerable state of conservation, so it is recommended to suspend their collection.

\section{Key words: Ethnobotany, flavonoids, Gentianaceae, Jalca, Quechua.}

${ }^{1}$ Programa de Raíces y Tubérculos Andinos, Facultad de Ciencias Agrarias, Universidad Nacional de Cajamarca, Cajamarca, Perú.

${ }^{2}$ Laboratorio de Biotecnología Vegetal, Universidad Nacional de Cajamarca, Cajamarca, Perú.

${ }^{3}$ Hersil S. A. Laboratorios Industriales Farmacéuticos, Lima, Perú.

${ }^{4}$ Carrera de Ingeniería Agroforestal, Universidad Científica del Sur, Lima, Perú.

${ }^{5}$ Unidad de Investigación en Nutrición, Salud, Alimentos Funcionales y Nutracéuticos, Universidad San Ignacio de Loyola, Lima, Perú. E-mail: ibest@usil.edu.pe 


\section{Introducción}

En el norte peruano existen más de 500 especies de plantas medicinales, que se ubican principalmente en la franja altoandina (Sánchez, 2014; Bussmann et Sharon, 2015; Galán de Mera et al., 2018), dentro de las cuales, se destacan varias del género Gentianella Moench (Gentianaceae). Este género se distribuye en regiones templadas y tropicales del mundo y comprende aproximadamente 250 especies, de las cuales 104 se registran en Perú (Castillo et Pringle, 2018). Se calcula que al menos 60 especies son endémicas de Perú (Castillo et al., 2006). La franja comprendida por encima de 2700 m s.n.m., correspondiente a las provincias de Cajamarca, Hualgayoc y Celendín (Región Cajamarca, Perú) resulta importante por su alta diversidad de plantas medicinales (Sánchez, 2014). Sin embargo, esta franja ha sido muy intervenida en las últimas décadas, principalmente por la agricultura, ganadería y minería. Las concesiones mineras para estas tres provincias cubren el $66 \%, 94 \%$ y $42 \%$ de sus territorios, respectivamente (Grupo Propuesta Ciudadana, 2014); constituyendo el principal factor de riesgo, por las explotaciones a tajo abierto que destruyen las áreas naturales. En el área del presente estudio, se han identificado ocho especies de Gentianella, de las diez reportadas para la Región Cajamarca: $G$. graminea (Kunth) Fabris ("chinchimalí", "sumarán", "corpushuay"), G. dianthoides (Kunth) Fabris ex J. S. Pringle ("sumarán anaranjado", "amargón anaranjado", "corpushay anaranjado", "campanilla", "genciana"), G. crassicaulis J. S. Pringle ("amargón morado", "corpushay morado", "corpushay hembra", "violeta genciana"), G. bicolor (Wedd.) Fabris ex J. S. Pringle ("corpushayta", "flor de cristo", "mashca mashca", "hórnamo de león”), G. raimondiana (Wedd.) J. S. Pringle, G. uberula J. S. Pringle y G. arenarioides (Gilg) J. S. Pringle, G. sanchezii J. S. Pringle (Pringle, 2008; Seminario et Sánchez, 2010; Bussmann et Sharon, 2015; Galán de Mera et al., 2018; Castillo et al., 2019). Las primeras cuatro especies son las más importantes por su uso tradicional y volumen de extracción para abastecer a los mercados locales, así como de la costa de Perú. Éstas y otras especies de
Gentianella [G. nitida Griseb., G. alborosea (Gilg) Fabris] son conocidas comercialmente como "hercampure" o "hercampuri" (Huamán et al., 2015; Carbonel et al., 2016). En la medicina tradicional y en el mercado se reconoce que los hercampures contienen principios amargos y se recomiendan para tratamiento de la gripe, diabetes, colesterol alto, obesidad, trastornos del hígado, detoxificación de la sangre, dolores estomacales, gastritis, afecciones de la piel (acné), dolores postparto, artritis, reumatismo así como estimulantes del apetito (Seminario et Sánchez, 2010; Bussmann et Sharon, 2015). Por su uso en medicina tradicional y como insumo para la elaboración de medicamentos, existe una incrementada recolección de estas especies.

Estas especies contienen polifenoles (del tipo flavonoides), xantonas, peptinas, saponinas, triterpenos secoiridoides, sesquiterpenoides, esteroles, taninos, leucoantocianinas y aminoácidos libres (Huamán et al., 2015; Carbonel et al., 2016). Los flavonoides son compuestos sintetizados en las plantas por la vía del fenilpropanoide, con propiedades antioxidantes, antiinflamatorias, antimutagénicas y anticarcinogénicas, con capacidad para regular sistemas enzimáticos y comportarse a su vez como prebióticos (Quiñones et al., 2012; Carbonel et al., 2016; De Paula et al., 2018). Para propiciar la conservación y uso sostenible de estas especies se requiere el conocimiento de la biología y ecología en su hábitat natural, como base para su domesticación y cultivo. El objetivo del presente estudio fue describir la biología y ecología de cuatro especies de Gentianella recolectadas para el mercado en las provincias de Cajamarca, Hualgayoc y Celendín procedentes de la Región Cajamarca, Perú; con especial énfasis en sus características productivas y su contenido de flavonoides totales.

\section{Materiales y Métodos}

\section{Área de estudio y especies involucradas}

La investigación se realizó entre mayo 2018 y septiembre 2019 (en 15 salidas de campo), en las regiones Quechua y Jalca (Pulgar Vidal, 1996) de las provincias de Hualgayoc, Celendín 
y Cajamarca de la Región Cajamarca, Perú; entre los 2700 y 4100 m s.n.m., donde se ubicaron las cuatro especies en estudio: Gentianella graminea, G. dianthoides, G. crassicaulis y $G$. bicolor.

Se tomaron como base los registros y colectas de Gentianella del Herbario Isidoro Sánchez Vega (CPUN) de la Universidad Nacional de Cajamarca y estudios previos realizados (Seminario et Sánchez, 2010). En las exploraciones de campo, se contó con el apoyo de personas de cada zona para localizar las especies con mayor población por superficie ocupada y densidad poblacional. Se colectaron muestras para el herbario de las especies en estudio, así como de sus especies asociadas. Las muestras fueron acondicionadas para su identificación, siguiendo la metodología de Rodríguez et Rojas (2006). La identificación de especies se realizó mediante comparación con los ejemplares identificados y depositados en el herbario CPUN de la Universidad Nacional de Cajamarca, según Pringle (2008). La actualización de la nomenclatura se realizó de acuerdo con The Plant List (2019) y Tropicos (2019). El material botánico identificado y etiquetado fue depositado en el herbario CPUN (Serie WSC 1-42).

\section{Biología de las especies en estado silvestre}

Morfología. Con plantas frescas de cada una de las especies evaluadas, se describieron algunas características de estas plantas, con especial énfasis en aspectos no considerados en la literatura. En 10 plantas tomadas al azar, se evaluó el número de frutos por planta, número de semillas por fruto, diámetro y peso de 1000 semillas.

Fenología y regeneración natural de las poblaciones. Se tomaron en cuenta poblaciones representativas, tomando en consideración la distribución, abundancia de cada especie y accesibilidad, pues las áreas más cercanas a las explotaciones mineras no fueron accesibles. Las poblaciones se localizaron en las comunidades de Sendamal y Coimolache para Gentianella graminea, Cumullca y Coimolache para $G$. dianthoides, Cumullca para G. crassicaulis, así como Sangal y Frutillo bajo para G. bicolor. Durante 12 meses, se hizo un seguimiento del desarrollo fenológico de cada especie, con énfasis en las etapas vegetativa y reproductiva, lo que permitió describir el ciclo anual de las poblaciones silvestres. La regeneración natural de las poblaciones se evaluó mediante el conteo del número de plántulas alrededor de las plantas (G. graminea y $G$. crassicaulis) o plantas adultas $(G$. dianthoides y $G$. bicolor) siguiendo la metodología utilizada para estudiar la regeneración de Valeriana pilosa en la Jalca de Huanico (Seminario-Cunya et al., 2016). La evaluación se hizo en 20 parcelas de $1 \mathrm{~m}^{2}$, seleccionadas al azar. Las plántulas presentes en estas áreas provienen de las semillas que caen al suelo en cada temporada de fructificación.

Germinación de semillas en laboratorio. Se realizó con semillas de 8 semanas de edad postcosecha y en 2 repeticiones de 100 semillas cada una. Las semillas fueron colocadas en placas Petri, conteniendo láminas de papel absorbente húmedo (con agua destilada) y se mantuvieron con luz y temperatura de laboratorio $\left(19^{\circ} \mathrm{C}\right)$ durante 60 días. Se evaluó el poder germinativo (número total de semillas germinadas), velocidad (tiempo necesario para que germine el $50 \%$ de semillas, con relación al total de semillas germinadas) y uniformidad de la germinación (tiempo entre las primeras y últimas semillas germinadas) (Pérez-Martínez et al., 2014a).

Determinación de la materia seca de las plantas. La materia seca de las plantas en estado de recolección se determinó tomando una muestra aleatoria de 30 plantas en plena floración. Para ello, las plantas fueron divididas en partes aérea y subterránea. Dichas secciones se pesaron y se colocaron en una estufa a $65^{\circ} \mathrm{C}$ hasta peso constante. El porcentaje de materia seca fue calculado tomando como referencia el peso fresco mediante la siguiente ecuación:

$$
\% \text { materia seca }=\frac{\text { peso fresco }}{\text { peso seco }} \times 100
$$

Flavonoides totales. Debido a la disponibilidad del material vegetal, la evaluación del contenido de flavonoides totales se realizó en tres especies ( $G$. graminea, $G$. dianthoides y G. crassicaulis). Los extractos se obtuvieron de hojas y tallos secos con diferentes concentraciones de etanol $(50 \%, 80 \%$ y $96 \%)$, 
diluidos $1 / 5(\mathrm{p} / \mathrm{v})$, en agitación constante, durante 3 días. Posteriormente, fueron filtrados con papel filtro Whatman $\mathrm{N}^{\circ} 2$. Los extractos secos se obtuvieron mediante concentración en rotavapor a $40{ }^{\circ} \mathrm{C}$ por 2 horas, seguido de secado en estufa a $40{ }^{\circ} \mathrm{C}$ durante 24 horas. Los flavonoides totales fueron cuantificados según el método de Wolfe et Liu (2013), con algunas modificaciones. Brevemente, a cada $250 \mu 1$ del extracto, se agregó $125 \mu 1$ de agua destilada y $75 \mu 1$ de nitrito de sodio al 5\%. Esta mezcla se dejó reposar durante 5 minutos, y se adicionó $150 \mu \mathrm{l}$ de tricloruro de aluminio al $10 \%$. Después de 6 minutos, se añadió 0,5 $\mathrm{ml}$ de hidróxido de sodio $2 \mathrm{M}$ y $275 \mu \mathrm{l}$ de agua destilada. Finalmente, se midió la absorbancia a $510 \mathrm{~nm}$, y se comparó con una curva estándar, utilizando diferentes concentraciones de catequina. El contenido de flavonoides totales fue expresado en miligramos equivalentes de catequina/gramo extracto seco (mg CE/g extracto seco).

\section{Ecología de las poblaciones}

Distribución y parámetros ambientales para las especies estudiadas. En los recorridos de campo, se ubicaron las poblaciones de cada especie a evaluarse, las cuales fueron georreferenciadas para elaborar un mapa de distribución distrital. En el hábitat de cada especie seleccionada, se describieron aspectos de la fisiografía y se tomaron muestras de suelo, para el análisis de textura, $\mathrm{pH}$, materia orgánica, fósforo disponible y potasio disponible (EEBIINIA, 2018). Se tomaron como referencia los datos de precipitación mensual y la temperatura diaria de una estación tipo para las especies de Jalca y otra para las especies de Quechua (ante la falta de estaciones meteorológicas en cada lugar): Estación Meteorológica de Huanico (3708 m s.n.m.) para G. graminea, $G$. dianthoides y G. crassicaulis (Asociación Civil para la Investigación y Desarrollo Forestal, 1995) y Estación Meteorológica del distrito de Jesús (2700 m s.n.m.) para G. bicolor (Climatedata.org, 2019).

Densidad de poblaciones, especies asociadas e indices de diversidad alfa. Con el propósito de determinar la densidad poblacional (en plantas/ $\mathrm{m}^{2}$, según la especie), la frecuencia relativa (Fr, $\%)$ y la abundancia relativa (Ar, \%) de cada especie, se contaron las especies y el número de individuos por especie (incluyendo las especies asociadas) en 20 puntos tomados al azar y en parcelas de $1 \mathrm{~m}^{2}$ de área (Mostacedo et Fredericksen, 2000), en las poblaciones seleccionadas. Asimismo, utilizando las fórmulas descritas por Mateucci et Colma (1982), se calcularon los índices de diversidad, equidad de Shannon-Wiener $\left(H^{\prime}\right)$ y dominancia de Simpson $(\lambda)$ (Magurran, 2004; Moreno, 2001).

\section{Análisis estadístico}

Los datos se presentan con los valores promedios, desviación estándar (DE), siendo analizados mediante el programa SPSS para Windows versión 24.0 (SPSS, Inc., Chicago, IL, USA). Se realizó un análisis de covarianza (ANCOVA) para evaluar el impacto de los días transcurridos para la toma de datos sobre el poder germinativo, uniformidad y velocidad de germinación. Un análisis de varianza (ANOVA) seguido de la Prueba de Comparación Múltiple de Tukey fue utilizada para evaluar diferencias en el porcentaje de materia seca y niveles de flavonoides totales. Los resultados fueron considerados significativos a un $p<0,05$.

\section{Resultados}

Biología de las cuatro especies de Gentianella de la Región Cajamarca, Perú

Morfología de las plantas. A continuación se presenta una descripción de las especies, de acuerdo a los datos recogidos en el presente estudio.

Gentianella graminea (Kunth) Fabris (Fig. 1). Es una hierba perenne de 15 a $22 \mathrm{~cm}$ de alt. De hojas verdes, botón floral de color blancopúrpura. Las flores (en cima corimbiforme terminal) blancas, campanuladas con cinco sépalos fusionados en forma de un tubo corto de color verde claro, pétalos unidos en la base. Filamentos estaminales amarillentos y anteras marrones. La cápsula de color marrón oscuro, de $16 \mathrm{~mm}$ de long. y de $2 \mathrm{~mm}$ de 

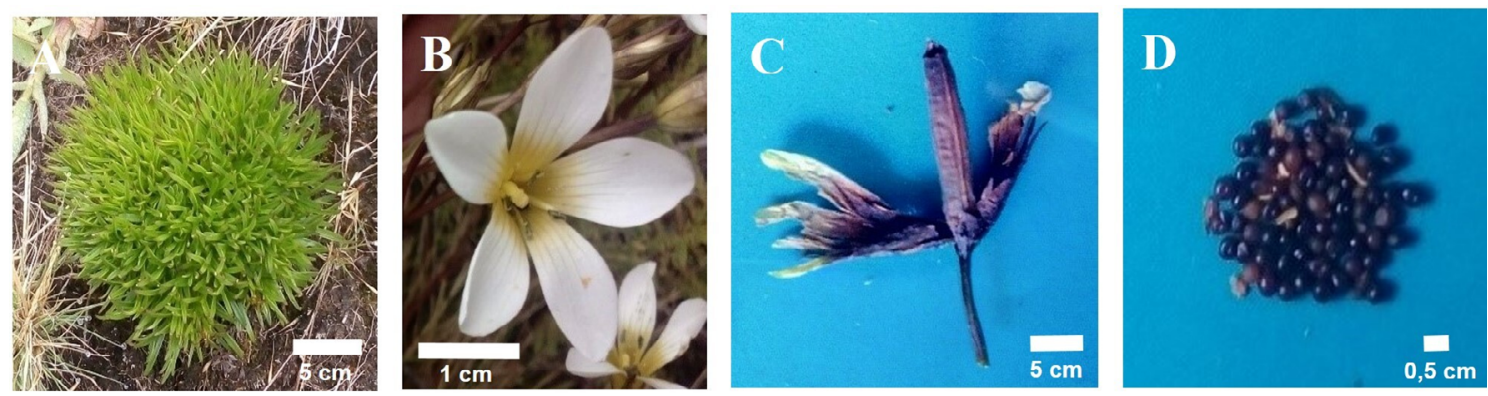

Fig.1. Gentianella graminea. A: Planta. B: Flor. C: Fruto. D: Semillas.

Fig. 1. Gentianella graminea. A: Plant. B: Flower. C: Fruit. D: Seeds.

diám. Las semillas marrones, lisas y esféricas, de $0,5 \mathrm{~mm}$ de diám. Cada fruto contenía en promedio 17 semillas. Mil semillas pesaron $180 \mathrm{mg}$.

Gentianella dianthoides (Kunth) Fabris ex Pringle (Fig. 2). Es una hierba anual de 35 $\mathrm{cm}$ de alt. Hojas verde-amarillentas. Las flores (en cima terminal) rojo-anaranjadas, campanuladas, cinco sépalos unidos, cinco pétalos, estambres cremosos y anteras marrones. La cápsula color marrón claro de $20 \mathrm{~mm}$ de long. y $3 \mathrm{~mm}$ de diám. Cada planta produjo de 3 a 30 frutos. Se encontraron en promedio 32 semillas por fruto y hasta 960 semillas por planta. Las semillas son esféricas, negras, de $0,5 \mathrm{~mm}$ de diám. Mil semillas pesaron $170 \mathrm{mg}$.

Gentianella crassicaulis J. S. Pringle (Fig. 3). Es una hierba perenne de mayor tamaño que las especies anteriores, alcanza hasta $45 \mathrm{~cm}$ de alt. Hojas verdes claro con borde morado, tallo con entrenudos cortos en la base. Las flores (en cima terminal umbeliforme) púrpuras, campanuladas, con tres ejes florales por planta. La cápsula de $22 \mathrm{~mm}$ de long. y $3 \mathrm{~mm}$ de diám. Cada planta produjo de 8 a 60 frutos y cada fruto contenía 12 semillas en promedio. Se encontraron hasta 720 semillas por planta. Las semillas marrones, elipsoideas, de $0,5 \mathrm{~mm}$ de diám. Mil semillas pesaron $285 \mathrm{mg}$.

Gentianella bicolor (Wedd.) Fabris (Fig. 4). Es una hierba anual de $30 \mathrm{~cm}$ de alt., de hojas verde púrpura. La flor (en cima terminal umbeliforme), púrpuraazulada, campanulada, con cinco sépalos fusionados en un tubo corto de color morado claro, cinco pétalos, con cinco estambres cremosos. La cápsula de color marrón-amarillento, de $18 \mathrm{~mm}$ de long. y 3 $\mathrm{mm}$ de diám. En cada planta se encontraron de 3 a 16 frutos y cada fruto contenía, en promedio, 28 semillas. Cada planta produce hasta 450 semillas. Las semillas son lisas, esféricas y negras, de $0,5 \mathrm{~mm}$ de diám. Mil semillas pesaron $303 \mathrm{mg}$.
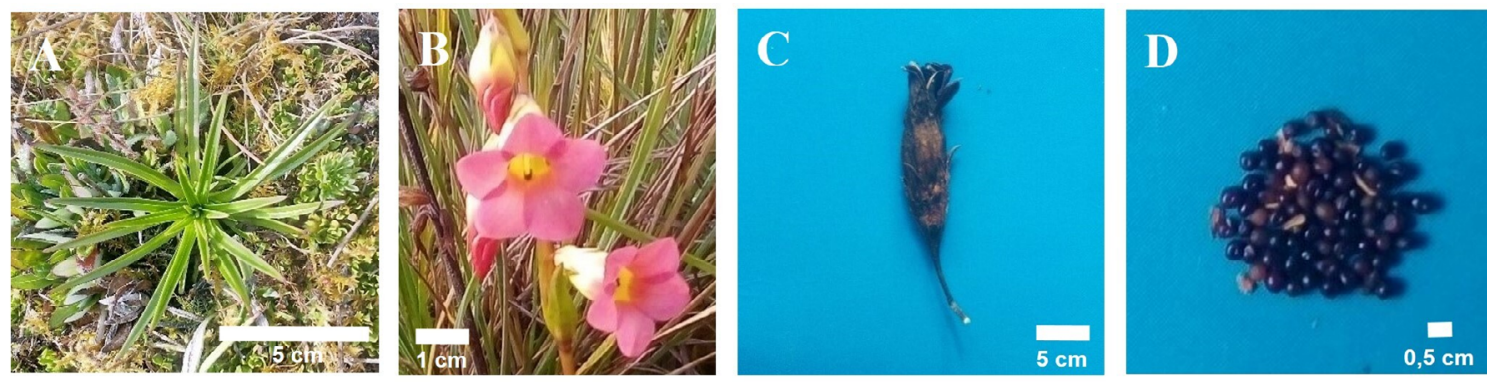

Fig. 2. Gentianella dianthoides. A: Planta. B: Inflorescencia. C: Fruto. D: Semillas.

Fig. 2. Gentianella dianthoides. A: Plant. B: Inflorescence. C: Fruit. D: Seeds. 

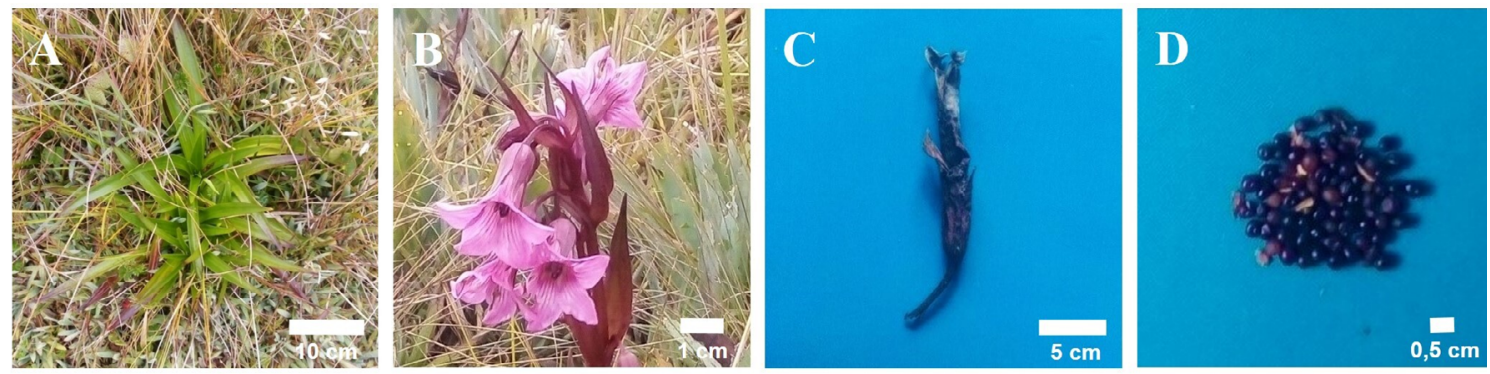

Fig. 3. Gentianella crassicaulis. A: Planta. B: Inflorescencia. C: Fruto. D: Semillas.

Fig. 3. Gentianella crassicaulis. A: Plant. B: Inflorescence. C: Fruit. D: Seeds.

Fenología y regeneración natural de las poblaciones. Gentianella dianthoides, $G$. crassicaulis y $G$. bicolor, estuvieron en estado vegetativo desde noviembre hasta abril. A partir de abril o inicios de mayo (coincidiendo con el inicio de la temporada de estiaje), se inició la fase reproductiva. En las tres especies, los procesos de floración y fructificación fueron progresivos, de modo que, en un momento determinado y sobre un mismo eje reproductivo, se encontraron flores, frutos y semillas en diferentes estados de desarrollo. En cambio, G. graminea, no presentó estadios fenológicos definidos, porque florece $\mathrm{y}$ fructifica todo el año, aunque con mayor intensidad entre junio y octubre. Gentianella graminea y $G$. crassicaulis se regeneran naturalmente de dos formas: (1) mediante la semilla producida en cada temporada y (2) mediante propagación vegetativa a través de los rizomas de las plantas adultas. Esto implica que una vez que termina la etapa reproductiva, los ejes reproductivos mueren y se generan nuevos brotes laterales que expondrán inflorescencias $\mathrm{y}$ frutos en la siguiente temporada. Esto es menos notable en $G$. graminea, en la cual se produce floración durante todo el año, debido a la presencia de brotes de diferente edad en cada planta. La regeneración de $G$. dianthoides y $G$. bicolor es solo a través de semilla. Por lo tanto, al final del ciclo las plantas mueren.

La regeneración natural por semilla en las cuatro especies fue baja: $2,3 \pm 1,2$ plántulas/ $\mathrm{m}^{2}$ en $G$. graminea; $3,5 \pm 1,2$ plántulas $/ \mathrm{m}^{2}$ en $G$. dianthoides; $1,2 \pm 0,4$ plántulas $/ \mathrm{m}^{2}$ en $G$. crassicaulis y $4,3 \pm 2,5$ plántulas $/ \mathrm{m}^{2}$ en $G$. bicolor.

Germinación de semillas en laboratorio. Las semillas de la temporada, después de ocho semanas postcosecha, empezaron a germinar en las placas Petri entre los 15 a 20 días de la siembra y continuaron germinando en número variable hasta la finalización el ensayo. El poder germinativo alcanzó los niveles más altos en $G$. dianthoides $(52 \%)$ y $G$. bicolor $(40 \%)$, en $G$. graminea y $G$. crassicaulis, los valores fueron de $16 \%$ y $10 \%$, respectivamente (Fig. 5A). Gentianella dianthoides presentó la mayor
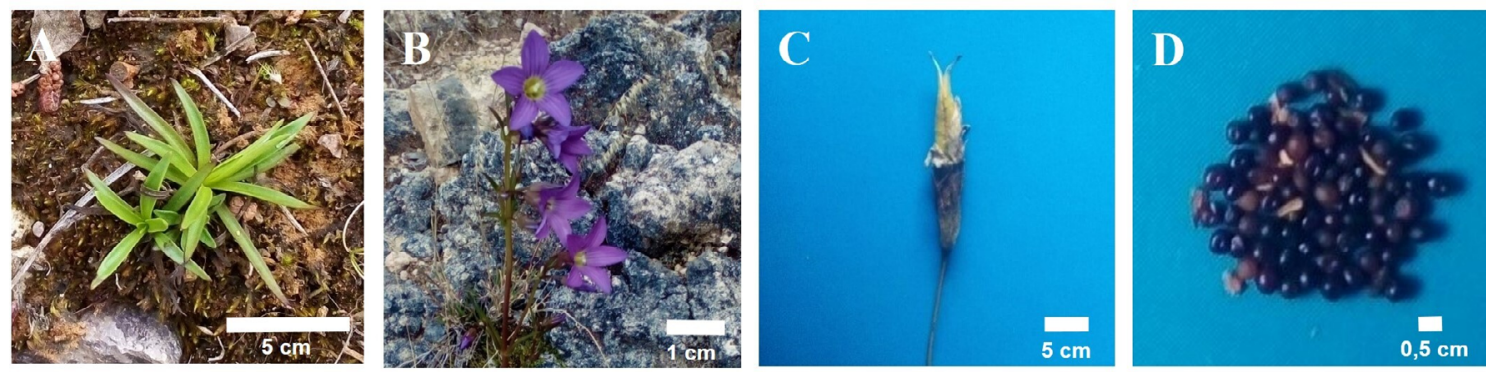

Fig. 4. Gentianella bicolor. A: Planta. B: Inflorescencia. C: Fruto. D: Semillas.

Fig. 4. Gentianella bicolor. A: Plant. B: Inflorescence. C: Fruit. D: Seeds. 
velocidad de germinación, el 50\% de las semillas germinó a los 25 días. En cambio, en G. bicolor, el $50 \%$ de semillas germinadas se alcanzó a los 30 días. Estas últimas especies presentaron la misma falta de uniformidad de germinación, obteniéndose un $80 \%$ de germinación en $70 \%$ del tiempo (Fig. 5B). En el análisis de covarianza (ANCOVA), se observó que los días transcurridos para la toma de datos influenció significativamente el poder germinativo, uniformidad y velocidad de germinación $(p<0,001)$ (datos no mostrados).

Determinación de la materia seca de las plantas. Se observó una variación de la materia seca entre la parte subterránea y aérea de la planta (Tabla 1). Esta variación se explica por la falta de uniformidad en las edades de los individuos evaluados en cada población (por ser silvestres, no se pudo conocer la edad, el único factor de uniformidad de los elementos de la muestra fue su estado de cosecha, como acostumbran los recolectores); asimismo, dichos individuos se desarrollaron en condiciones ecológicas heterogéneas. En las cuatro especies de Gentianella evaluadas, la parte subterránea y aérea fluctuó entre $18,18 \%$ a $53,33 \%$ y $14,42 \%$ a $56,52 \%$ de la materia seca, respectivamente. Gentianella dianthoides y G. bicolor presentaron un significativo mayor porcentaje de materia seca en la parte subterránea $(p<0,05)$, mientras que $G$. graminea presentó un porcentaje significativamente mayor de materia seca en la parte aérea comparado con las demás especies
Tabla 1. Porcentaje de materia seca de la planta en las especies de Gentianella analizadas: valores promedios \pm desviación estándar $(\overline{\mathrm{X}} \pm \mathrm{DE})$.

Table 1. Percentage of dry matter of the plant in the Gentianella species analyzed: mean values \pm standard deviation $(\overline{\mathrm{X}} \pm \mathrm{SD})$

\begin{tabular}{|lcc|}
\hline \multicolumn{1}{|c}{ Especie } & \multicolumn{2}{c|}{$\%$ materia seca } \\
& Subterránea & Aérea \\
G. graminea & $30,83 \pm 9,86^{\mathrm{b}}$ & $37,84 \pm 5,42^{\mathrm{a}}$ \\
G. dianthoides & $38,75 \pm 8,47^{\mathrm{a}}$ & $31,83 \pm 5,80^{\mathrm{b}}$ \\
G. bicolor & $40,04 \pm 9,18^{\mathrm{a}}$ & $30,25 \pm 7,93^{\mathrm{b}}$ \\
G. crassicaulis & $28,98 \pm 5,59^{\mathrm{b}}$ & $28,93 \pm 6,91^{\mathrm{b}}$ \\
\hline
\end{tabular}

Valores en la misma columna con letras diferentes (a-b) difieren significativamente $(p<0,05)$ (ANOVA y Prueba de comparación múltiple de Tukey).

analizadas $(p<0,05)$. Por su gran tamaño, $G$. crassicaulis, rindió entre 5 a 16 veces más materia seca que las otras especies (datos no mostrados).

Flavonoides totales. En todas las especies evaluadas, el contenido de flavonoides totales fue significativamente mayor en la extracción con $96 \%$ etanol, respecto a las extracciones con $50 \%$ y $80 \%$ etanol $(p<0,05)$. Gentianella graminea presentó niveles de flavonoides totales significativamente mayores en hoja y tallo respecto a $G$. dianthoides y $G$. crassicaulis $(p$
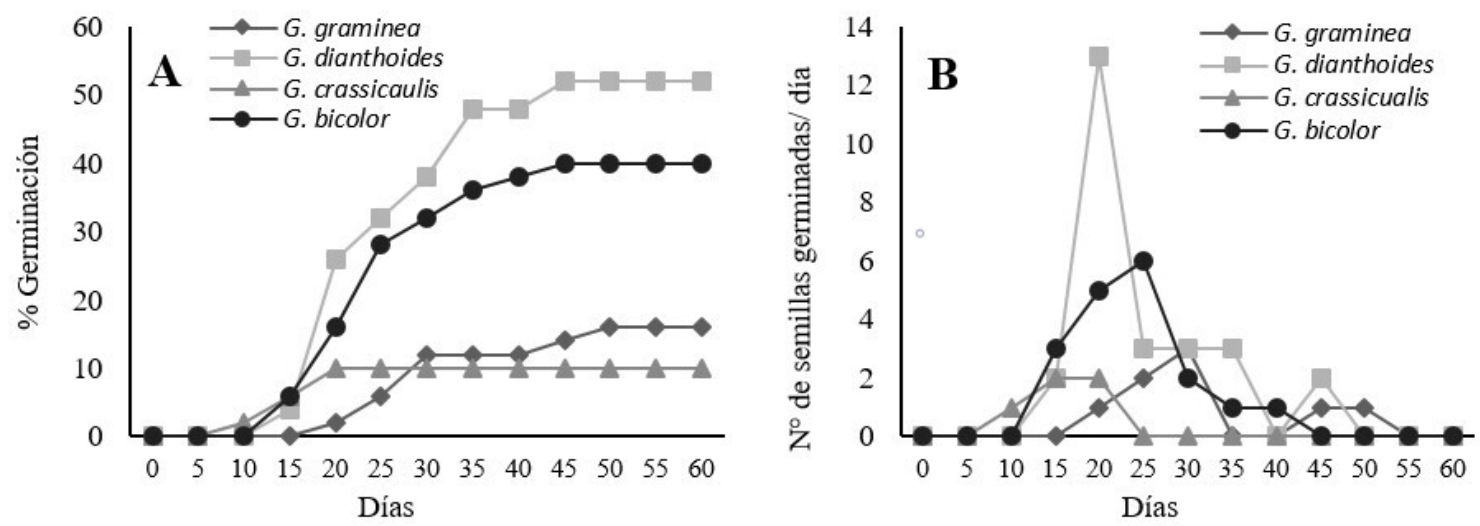

Fig. 5. Poder germinativo (A) y Uniformidad y velocidad de germinación (B) de las especies de Gentianella analizadas. Fig. 5. Germination power (A) and Uniformity and germination rate (B) of the Gentianella species analyzed. 
$<0,05)$. Asimismo, en todos los extractos con diferentes concentraciones de etanol $(50 \%$, $80 \%$ y $96 \%$ ), los niveles de flavonoides totales de las tres especies, fueron significativamente mayores en hoja respecto al tallo $(p<0,05)$ (Tabla 2).

\section{Ecología de las poblaciones de Gentianella en tres provincias de la Región Cajamarca}

Distribución y parámetros ambientales para las especies estudiadas. Las cuatro especies se distribuyeron en la parte más alta de 15 distritos de las tres provincias en estudio, en las regiones Quechua y Jalca, entre los 2700 a 4100 m s.n.m. Esta ubicación corresponde a los pisos mesotropical, supratropical y orotropical (Galán de Mera et al., 2019), con diferencias entre especies y provincias de estudio (Fig. 6). Gentianella graminea, se desarrolló en la Jalca, en espacios poco impactados por la actividad antropogénica, entre los 3500 a 4100 m s.n.m., en cerros y planicies, en suelos húmedos o secos. Gentianella dianthoides, se ubicó en la Jalca, en áreas cercanas a $G$. graminea, habita principalmente en faldas de los cerros, entre 3800 a 4100 m s.n.m., donde la intensidad del viento es alta. Gentianella crassicaulis, es la especie de menor distribución, se desarrolló en la Jalca, entre los 3800 a 4000 m s.n.m., en áreas pequeñas, en suelos de poca pendiente y húmedos o pantanosos. En cambio, G. bicolor es típica de la Quechua, ubicada entre los 2700 y 3200 m s.n.m. Habita laderas y colinas de suelos secos y pedregosos. Los suelos donde habitan las especies de Jalca son de textura intermedia, de alta acidez, alto contenido de materia orgánica, alto contenido de fósforo disponible y contenido entre medio y alto de potasio disponible. En cambio, la especie de Quechua (G. bicolor), habita suelos de textura pesada, $\mathrm{pH}$ neutro, contenido medio de materia orgánica, contenido bajo de fósforo disponible y alto contenido de potasio disponible (Tabla 3 ).

Las variables climáticas en la Estación Meteorológica de Huanico (3708 m s.n.m.), típica para las especies de Jalca, indicaron temperaturas máximas de $11,9 \pm 0,6{ }^{\circ} \mathrm{C}$, mínimas de $2,4 \pm 2,0{ }^{\circ} \mathrm{C}$ y medias de $7,2 \pm$ $1,2{ }^{\circ} \mathrm{C}$; humedad relativa de $72,0 \pm 2,0 \%$ y $858 \mathrm{~mm}$ de lluvia anual. La precipitación mensual y la temperatura diaria promedio, se muestran en la Fig. 7A. Por otro lado, en los hábitats de la Quechua, la temperatura diaria promedio diaria osciló entre 13,1 a $15,4{ }^{\circ} \mathrm{C}$ y la precipitación promedio anual fue de $827 \mathrm{~mm}$. La precipitación mensual y la temperatura diaria promedio se muestran en la Fig. 7B.

Densidad de poblaciones, especies asociadas $e$ indices de diversidad alfa. Las densidades de las especies en estudio fueron: $2,5 \pm 1,0$ plantas $/ \mathrm{m}^{2}$ para G. graminea; 2,0 $\pm 0,7$ plantas/ $\mathrm{m}^{2}$ para $G$. dianthoides; $1,4 \pm 0,5$ plantas $/ \mathrm{m}^{2}$ para $G$. crassicaulis y $2,0 \pm 0,9$ plantas $/ \mathrm{m}^{2}$ para G. bicolor. Las tres primeras, ubicadas en la

Tabla 2. Contenido de flavonoides totales ( $\mu$ g equivalentes de catequina/mg extracto seco) en hoja y tallo a partir de extractos con diferentes concentraciones de etanol $(50 \%, 80 \%$ y $96 \%)$ en las especies de Gentianella analizadas: valores promedios \pm desviación estándar $(\overline{\mathrm{X}} \pm \mathrm{DE})$.

Table 2. Total flavonoid content ( $\mu \mathrm{g}$ catechin equivalents / $\mathrm{mg}$ dry extract) in leaf and stem from extracts with different ethanol concentrations $(50 \%, 80 \%$ and $96 \%)$ in the Gentianella species analyzed: mean values \pm standard deviation ( $\overline{\mathrm{X}} \pm \mathrm{SD}$ ).

\begin{tabular}{|crrrrrrrrr|}
\hline Parte & \multicolumn{3}{c}{ G. graminea } & \multicolumn{4}{c}{ G. dianthoides } & \multicolumn{3}{c|}{ G. crassicaulis } \\
$\begin{array}{c}\text { \% } \\
\text { etanol }\end{array}$ & 50 & 80 & 96 & 50 & 80 & 96 & 50 & 80 & 96 \\
Hoja & 27,32 & 31,69 & 39,00 & 29,11 & 26,81 & 35,15 & 30,27 & 32,53 & 33,06 \\
& $\pm 0,62^{\mathrm{e}, \mathrm{f}}$ & $\pm 0,63^{\mathrm{c}, \mathrm{d}}$ & $\pm 0,48^{\mathrm{a}}$ & $\pm 1,16^{\mathrm{d}, \mathrm{e}, \mathrm{f}}$ & $\pm 0,91^{\mathrm{f}}$ & $\pm 2,51^{\mathrm{b}}$ & $\pm 0,86^{\mathrm{c}, \mathrm{d}, \mathrm{e}}$ & $\pm 0,47^{\mathrm{b}, \mathrm{c}}$ & $\pm 0,67^{\mathrm{b}, \mathrm{c}}$ \\
Tallo & 18,30 & 20,81 & 27,18 & 9,86 & 12,53 & 16,95 & 12,55 & 14,85 & 16,77 \\
& $\pm 0,69^{\mathrm{c}}$ & $\pm 0,38^{\mathrm{b}}$ & $\pm 0,94^{\mathrm{a}}$ & $\pm 0,41^{\mathrm{f}}$ & $\pm 0,25^{\mathrm{e}}$ & $\pm 1,07^{\mathrm{c}}$ & $\pm 0,52^{\mathrm{e}}$ & $\pm 1,00^{\mathrm{d}}$ & $\pm 0,43^{\mathrm{c}, \mathrm{d}}$ \\
\hline
\end{tabular}

Valores en la misma fila con letras diferentes (a-f) difieren significativamente $(p<0,05)$ (ANOVA y Prueba de comparación múltiple de Tukey). 

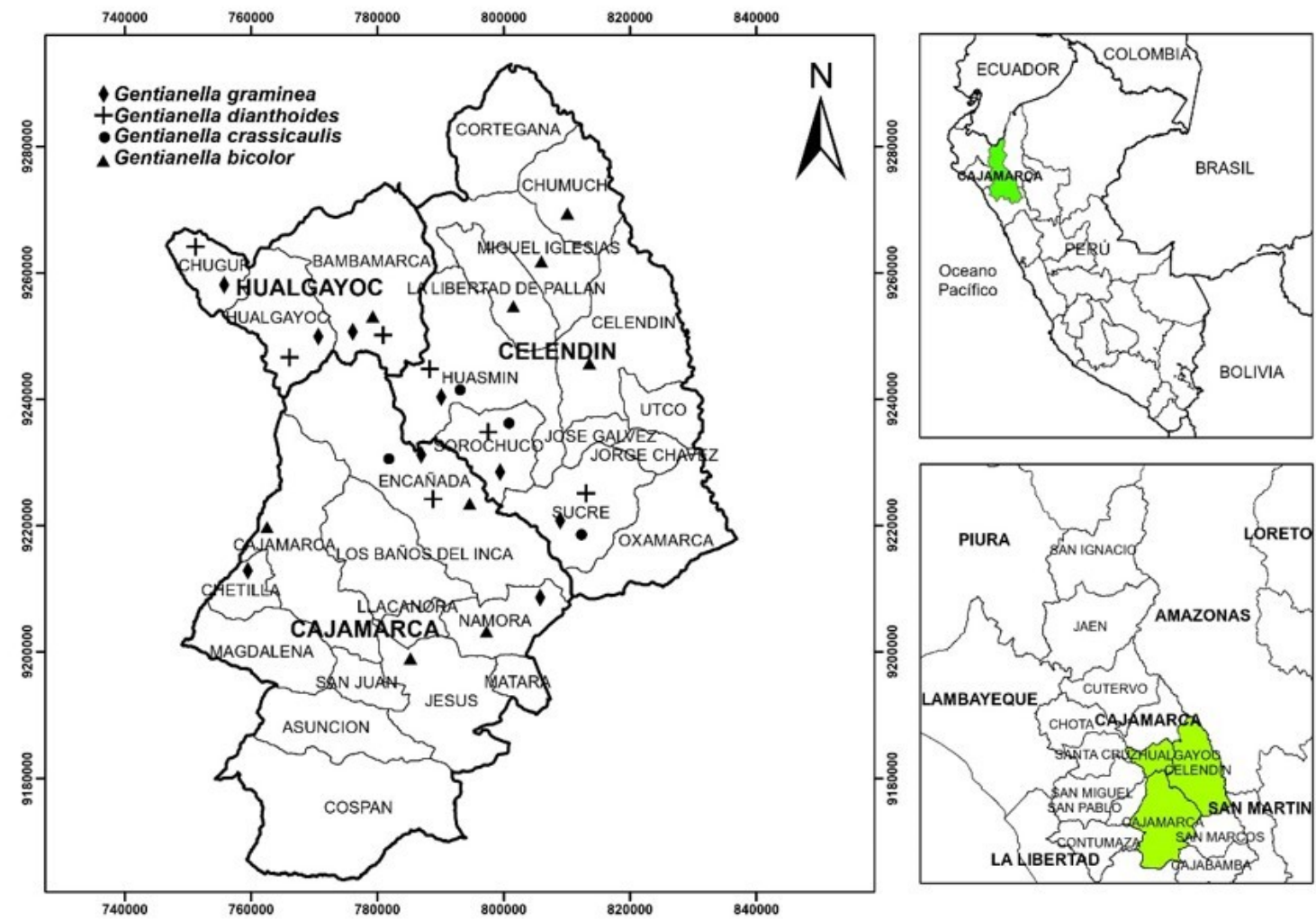

Fig. 6. Distribución de 4 especies de Gentianella en las provincias de Hualgayoc, Celendín y Cajamarca, Región Cajamarca, Perú.

Fig. 6. Distribution of 4 species of Gentianella in the provinces of Hualgayoc, Celendín and Cajamarca, Cajamarca Region, Peru.

Jalca están asociadas con 18 (G. crassicaulis) a 30 (G. graminea) especies pertenecientes a diez familias, con predominio de Asteraceae, Poaceae y Caprifoliaceae. Del total de especies asociadas en la Jalca, 16 son también medicinales recolectadas para el mercado (Tabla 4). Gentianella bicolor está asociada a 17 especies, pertenecientes a ocho familias, con predominio de Poaceae y Asteraceae. Nueve de estas especies asociadas son también medicinales y

Tabla 3. Características físicas y químicas de los suelos habitados por las especies de Gentianella analizadas.

Table 3. Physical and chemical characteristics of the soils inhabited by the Gentianella species analyzed.

\begin{tabular}{|lllccc|}
\hline \multicolumn{1}{|c}{ Especie } & \multicolumn{1}{c}{ Textura } & pH & MO (\%) & $\begin{array}{c}\text { P disponible } \\
\text { (ppm) }\end{array}$ & $\begin{array}{c}\text { K disponible } \\
\text { (ppm) }\end{array}$ \\
\hline G. graminea & Franco & 3,9 & 5,7 & 35 & 180 \\
G. dianthoides & Franco-arenoso & 4,5 & 6,3 & 23 & 110 \\
G. crassicaulis & Franco-arenoso & 5,1 & 9,9 & 58 & 235 \\
G. bicolor & Franco-arcilloso & 7,3 & 3,4 & 6,7 & 345 \\
\hline
\end{tabular}

MO: Materia orgánica, P: Fósforo, K: Potasio. Fuente: Laboratorio de EEBI-INIA (2018). 

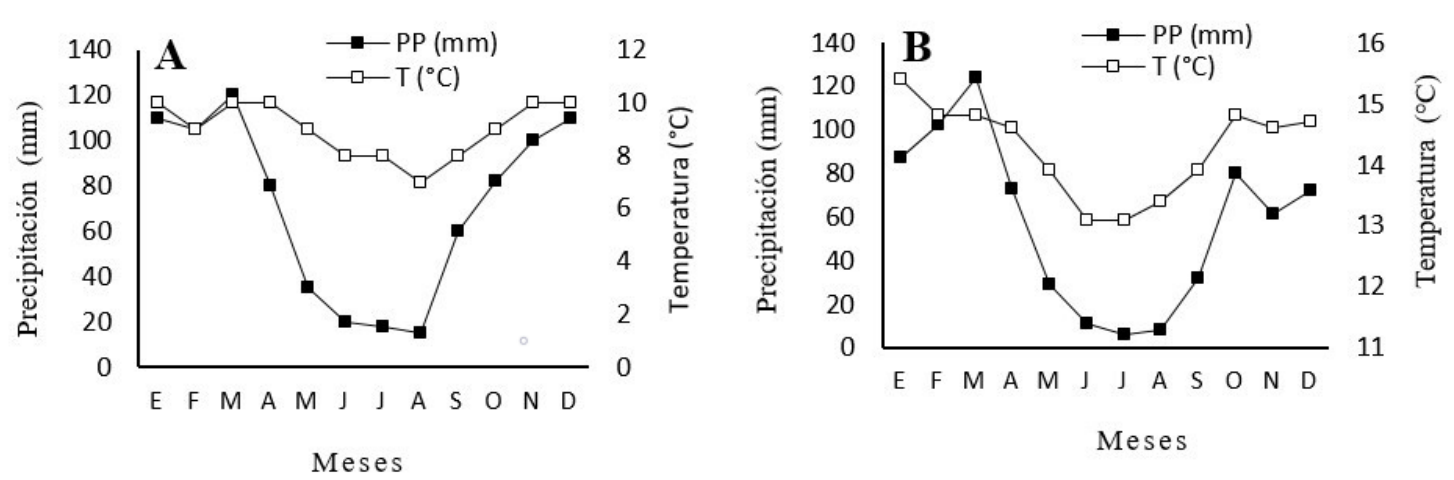

Fig. 7. Precipitación pluvial (PP, mm/mes) y temperatura promedio diaria $\left(\mathrm{T},{ }^{\circ} \mathrm{C}\right)$. A: Estación meteorológica de Huanico 3708 m s.n.m. (ADEFOR, 1995). B: Estación meteorológica de Jesús, Cajamarca - 2700 m s.n.m. (Climate-data.org, 2019). Fig. 7. Rainfall (PP, mm/month) and average daily temperature $\left(\mathrm{T},{ }^{\circ} \mathrm{C}\right)$. A: Huanico meteorological station - $3708 \mathrm{~m}$ a.s.1. (ADEFOR, 1995). B: Meteorological station of Jesús, Cajamarca - 2700 m a.s.1. (Climate-data.org, 2019).

recolectadas para la comercialización (Tabla 4). Ninguna de las especies en estudio es dominante en su respectivo hábitat. La frecuencia relativa y abundancia relativa fueron bajas, en relación a las especies con los valores más altos en su mismo hábitat (Tabla 5), correspondiendo a frecuencias absolutas de 3 a 6 registros, en el total de observaciones. Los valores del índice

Tabla 4. Especies asociadas a las especies de Gentianella procedentes de las provincias de Hualgayoc, Celendín y Cajamarca, Región Cajamarca, Perú.

Table 4. Species associated with Gentianella species from the provinces of Hualgayoc, Celendín and Cajamarca, Cajamarca Region, Peru.

Asociadas con G. graminea, G. dianthoides y G. crassicaulis Región Jalca

\section{Achyrocline alata (Kunth) DC., Achyrocline}

celosioides (Kunth) DC., Alchemilla orbiculata Ruiz \&

Pav.., Ageratina articulata (Sch. Bip. ex Hieron.) R. M.

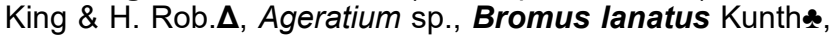

Calamagrostis tarmensis Pilg., Carex hebetata

Boott $\Delta$, Calamagrostis heterophylla (Wedd.) Pilg. $\diamond$,

Cerastium subspicatum Wedd. $\Delta$, Chenopodium sericeum Vitman,

Chaptalia cordata Hieron., Gentiana sedifolia Kunth,

Gnaphalium dombeyanum DC.*, Gnaphalium elegans Kunthn,

Hypericum laricifolium Juss. $\Delta$, Lolium multiflorum Lam., Luciliocline spathulifolia (Sagást. \& M. O. Dillon) M. O.

Dillon \& Sagást. $\Delta$, Oreomyrrhis andicola (Kunth) Endl. ex Hook. f $\square$, Oritrophium peruvianum (Lam.) Cuatrec. *,

Paranephelius uniflorus Poepp., Paronychia andina A. Gray口, Paspalum bonplandianum Flüggé, Pentacalia andicola (Turcz.) Cuatrec., Perezia pungens (Bonpl.) Less. $\Delta$,

Pernettya prostrata (Cav.) DC. $\Delta$, Phyllactis tenuifolia (Ruiz \& Pav.) Pers., Poa brevis Hitchc., Senecio canescens (Bonpl.) Cuatrec., Stipa ichu (Ruiz \& Pav.) Kunth , Trifolium repens L.•, Valeriana pilosa Ruiz \& Pav. Werneria nubigena Kunth.
Asociadas con G. bicolor Región Quechua

Populus L., Achyrocline celosioides (Kunth) DC., Agave americana L., Alternanthera macbridei Standl., Arcytophyllum ericoides (Willd. ex Roem. \& Schult.) Standl., Baccharis hutchisonii Cuatrec., Baccharis obtusifolia Kunth, Bromus lanatus Kunth, Calamagrostis heterophylla (Wedd.) Pilg., Eucalyptus globulus Labill., Lolium multiflorum Lam., Minthostachys mollis Griseb., Monnina conferta Ruiz \& Pav., Pennisetum clandestinum Hochst. ex Chiov., Poa brevis Hitchc., Rubus robustus C. Presl, Schizachyrium sanguineum (Retz.) Alston

Referencias: $\Delta$ Solo para G. graminea. • Solo para G. dianthoides. $\diamond$ Solo para G. crassicaulis. \& Para G. graminea y G. dianthoides. — Para G. graminea y G. crassicaulis. $\square$ Para G. dianthoides y G. crassicaulis. El resto son comunes para todas. En negrita se escriben las especies medicinales. 
Tabla 5. Frecuencia relativa (Fr) y abundancia relativa (Ar) de las especies de Gentianella analizadas y de sus dos especies asociadas con los valores más altos, en su hábitat respectivo.

Table 5. Relative frequency (Fr) and relative abundance (Ar) of the Gentianella species analyzed and of its two species associated with the highest values, in their respective habitat.

\begin{tabular}{|lclc|}
\hline \multicolumn{1}{|c}{ Especies } & Fr (\%) & \multicolumn{1}{c|}{ Especies } & Ar (\%) \\
G. graminea & 2,60 & G. graminea & 0,43 \\
Calamagrostis tarmensis & 10,60 & Paspalum bonplandianum & 22,10 \\
Paspalum bonpladianum & 6,60 & Werneria nubigena & 12,20 \\
G. dianthoides & 2,50 & G. dianthoides & 0,50 \\
Lolium multiflorum & 6,80 & Paspalum bonplandianum & 31,20 \\
Paspalum bonplandianum & 6,80 & Phyllactis tenuifolia & 9,70 \\
G. crassicaulis & 5,40 & G. crassicaulis & 0,70 \\
Calamagrostis tarmensis & 12,60 & Paspalum bonplandianum & 42,80 \\
Werneria nubigena & 11,70 & Werneria nubigena & 15,90 \\
G. bicolor & 5,00 & G. bicolor & 0,60 \\
Schizachyrium sanguineum & 17,70 & Schizachyrium sanguineum & 55,90 \\
Baccharis hutchisonii & 7,60 & Bromus lanatus & 12,00 \\
\hline
\end{tabular}

de Shannon-Wiener fueron intermedios, si se comparan con los valores máximos esperados, con diferencias entre las especies de Jalca y Quechua. Magurran (2004) y Vargas et Sánchez (2019), indican que los valores esperados son cero cuando solo existe una especie, bajo $(0,1-$ $2,9)$, medio $(3,0-4,4)$ y alto $(\geq 4,5)$. Por otro lado, los valores del índice de Simpson son bajos y en dos casos cercanos al valor mínimo esperado (Tabla 6).

Tabla 6. Índices de diversidad alfa en los hábitats de las especies de Gentianella analizadas.

Table 6. Alpha diversity indices in the habitats of the Gentianella species analyzed.

\begin{tabular}{|c|c|c|c|c|}
\hline Hábitat de & $\begin{array}{l}\text { Shannon-Wiener } \\
H^{\prime}=-\Sigma p i \text { In pi }\end{array}$ & $\begin{array}{l}\text { Rango } \\
0-\ln S\end{array}$ & $\begin{array}{l}\text { Simpson } \\
\lambda=\sum \mathbf{p i}^{2}\end{array}$ & $\begin{array}{r}\text { Rango } \\
0-(1-1 / S)\end{array}$ \\
\hline G. graminea & 2,70 & $0,00-3,40$ & 0,10 & $0,00-0,97$ \\
\hline G. dianthoides & 2,30 & $0,00-3,30$ & 0,17 & $0,00-0,96$ \\
\hline G. crassicaulis & 1,86 & $0,00-2,90$ & 0,25 & $0,00-0,94$ \\
\hline G. bicolor & 1,60 & $0,00-2,80$ & 0,35 & $0,00-0,94$ \\
\hline
\end{tabular}

\section{Discusión}

Las cuatro especies de Gentianella evaluadas son hierbas pequeñas, morfológicamente similares entre sí y con otras especies descritas previamente para Perú en general (Pringle,
2008, 2017a, 2017b). A excepción de $G$. graminea (la cual florece y fructifica todo el año), las tres especies restantes sincronizan sus fases fenológicas con las temporadas de estiaje y de lluvias; de modo que la floración y fructificación ocurren en los meses de estiaje y 
con bajas temperaturas. Este comportamiento es diferente al de otras especies medicinales de Jalca o Páramo, como Valeriana prionophylla (Contreras et Méndez-Estrada, 2014) y Valeriana pilosa (Seminario-Cunya et al., 2016), en las cuales la floración y fructificación suceden en los meses de mayor precipitación y temperatura (independientemente de la latitud). Las cuatro especies de Gentianella analizadas mostraron bajos valores de regeneración natural mediante semillas (1-4 plántulas $\left./ \mathrm{m}^{2}\right)$, en comparación a Valeriana pilosa, especie del mismo hábitat, en la cual, se encontró hasta 1124,8 plántulas $/ \mathrm{m}^{2}$ (Seminario-Cunya et al., 2016). Es decir, tienen baja capacidad para la restauración ecológica (Isuasty-Torres et al., 2014). Los factores involucrados pueden ser la poca producción de semilla, la densidad de la vegetación acompañante que impide la caída de las semillas al suelo y la pérdida de semillas y plántulas por el pastoreo. Estos factores deben analizarse para establecer estrategias o planes de manejo de las especies, con propósito de cosecha y conservación sostenibles (Vargas et Pérez-Martínez, 2014). Ninguna de las cuatro especies en estudio alcanzó un poder germinativo aceptable desde el punto de vista agronómico $(\geq 75 \%$ ) (Rao et al., 2007). Estos valores pueden deberse a diversas formas de dormancia y a la necesidad de requerimientos especiales para mejorar la germinación. Por ejemplo, en otra especie (Halenia major Wedd.) de la misma familia y también de Páramo, se encontró dormancia fisiológica (embrión inmaduro) y respondió a la estratificación y a temperaturas día/noche de $20{ }^{\circ} \mathrm{C} / 10{ }^{\circ} \mathrm{C}$ a $30{ }^{\circ} \mathrm{C} / 20{ }^{\circ} \mathrm{C}$. Además, en la evaluación de 13 especies de Páramo, la germinación varió de 18-20\% (Aragoa abietina Kunth) hasta 70-90\% (Puya trianae Baker) (Pérez-Martínez et al., 2014b). Dichas diferencias pueden explicarse también, por las características particulares de cada especie. En el caso de Gentianella graminea, las semillas son escasas y no maduran al mismo tiempo dentro del fruto. Asimismo, en un momento determinado, en la misma planta existen flores, frutos y semillas de diferente edad. En G. crassicaulis se forman muy pocas semillas, aunque son de maduración uniforme. En cambio, en $G$. dianthoides y $G$. bicolor existe mayor número de frutos y semillas y su maduración es más uniforme. La germinación no uniforme y escasa en especies silvestres, constituye una estrategia adaptativa frente a ambientes aleatorios y adversos. Dicha estrategia determina el tipo de banco de semillas necesario para la regeneración natural (Vargas et Pérez-Martínez, 2014). Desde el punto de vista comercial, a excepción de $G$. crassicaulis (que puede producir hasta $92 \mathrm{~g}$ de materia seca por planta), las otras tres especies son poco atractivas como proveedoras de materia prima para la industria. El cultivo tecnificado, puede mejorar esta condición en el mediano plazo, pero se necesita ensayar tratamientos específicos para mejorar la productividad, como se ha realizado en otras especies (MartínezArévalo, 2011). La biomasa subterránea en las cuatro especies fue poco significativa, de modo que la cosecha comercial dirigida a la parte aérea sería menos perjudicial para la conservación de las especies (OMS, 2003; Bussmann et Sharon, 2015). Esta información es importante, para evaluar los cambios en las plantas cultivadas y domesticadas (Raya-Pérez et al., 2010).

Gentianella graminea representó la mejor fuente de flavonoides con valores más altos en hoja. A su vez, las tres especies mostraron niveles mayores de flavonoides totales que el reportado por Carbonel et al. (2016) en el extracto acuoso de $G$. nitida de la Región Junín (Perú) correspondiente a 11,70 $\mu \mathrm{g}$ CE/ $\mathrm{mg}$ de extracto. Nuestros resultados fueron comparables con los niveles de flavonoides totales de extractos acuosos de 10 variedades de "té" [Camellia sinensis (L.) Kuntze], especie reconocida por su alto contenido de flavonoides. En estas variedades, los niveles de flavonoides totales variaron entre 17,70 a $50,12 \mu \mathrm{g} \mathrm{CE} / \mathrm{mg}$ extracto seco (Nibir et al., 2017).

Las cuatro especies evaluadas se ubican en ecosistemas frágiles (Jalca y Quechua) y en alto riesgo, debido a procesos acelerados de destrucción por la agricultura, ganadería y minería (Sánchez, 2014). Si se considera que en promedio $67 \%$ de estos territorios están concesionados a la minería; esta actividad es su mayor amenaza y es necesario proponer el cultivo tecnificado de Gentianella en otras áreas ecológicamente similares. 
Uno de los problemas a superar es la alta concentración de aluminio (de hasta $7 \mathrm{meq} / 100$ g) en los suelos de la Jalca (Nazar et Alva, 2015; Seminario-Cunya et al., 2016), elemento que constituye un factor limitante para el desarrollo de las plantas.

Las cuatro especies fueron de distribución escasa y baja densidad poblacional, debido a que sus hábitats son altamente intervenidos. Los recolectores indican que las poblaciones han disminuido considerablemente en las últimas décadas. En consecuencia, se estima que un recolector entrenado para reunir una arroba $(11,50 \mathrm{~kg}$, aproximadamente) de producto fresco -cantidad significativa desde el punto de vista económico del recolector- demanda de 30 minutos (G. crassicaulis), tres horas ( $G$. graminea) y cuatro horas $(G$. dianthoides y $G$. bicolor), respectivamente. Por otro lado, $G$. dianthoides y $G$. crassicaulis, deben ser motivo de atención mediante estrategias de conservación, dado su estado vulnerable en el Perú, según criterios de la UICN (Castillo et al., 2006).

Las cuatro especies en estudio viven asociadas a 42 especies, de las cuales 25 son también medicinales, recolectadas para el mercado (Seminario et Sánchez, 2010; Sánchez, 2014), lo que implica que todas están amenazadas y las medidas de conservación deberían tomar en cuenta esta situación. La diversidad fue ligeramente superior en los hábitats de Jalca respecto a la Quechua, lo cual se explica por las características de los hábitats: en la Quechua, los suelos son secos, poco profundos y con bajo contenido de materia orgánica. Por el contrario, en la Jalca, los suelos son más profundos y con mayor humedad durante todo el año. También se explica porque los hábitats de la Quechua son más intervenidos y con menos vegetación silvestre. Por otro lado, se observa que mientras más bajo es el índice de Simpson (hábitats de Jalca), más diversa es la comunidad (Moreno, 2001). Probablemente, su valor estuvo fuertemente influenciado por las especies dominantes, Calamagrostis tarmensis, Paspalum bonplandianum, Phyllactis tenuifolia y Werneria nubigena, en la Jalca y Schizachyrium sanguineum en la Quechua. Nuestros resultados permiten sugerir medidas de conservación como la zonificación de actividades, la declaración de áreas de reserva y planes de manejo concertado entre recolectores, comerciantes y los organismos oficiales.

\section{Agradecimientos}

Este estudio fue parte del proyecto "Nutracéutico con alto contenido de fructoligosacáridos y compuestos fenólicos como coadyuvante en el tratamiento de la obesidad elaborado a partir de hercampuri (Gentianella alborosea) y yacón (Smallanthus sonchifolius), procedentes del departamento de Cajamarca" financiado por INNOVATE PERU (Contrato 685-INNOVATEPERUPICE1-2017), realizado en convenio entre la Universidad Nacional de Cajamarca y Representaciones FRANCAR. Agradecemos al Ingeniero Juan Montoya Quino (Curador del herbario CPUN), por su colaboración en la identificación de las especies.

\section{Bibliografía}

ASOCIACIÓN CIVIL PARA LA INVESTIGACIÓN Y DESARROLlo Forestal. (1995). Registro de 19 estaciones meteorológicas. Boletín Meteorológico, ADEFOR, Cajamarca, Perú.

Bussmann, R. W. \& Sharon, D. (2015). Plantas medicinales de los Andes y la Amazonía-La flora mágica y medicinal del Norte del Perú. Centro William L. Brown-Jardín Botánico de Missouri, Trujillo, Perú.

Carbonel, K., Suárez, S. \& Arnao, A. (2016). Características fisicoquímicas y capacidad antioxidante in vitro del extracto de Gentianella nitida. Anales de la Facultad de Medicina 77: 333 337. https://doi.org/10.15381/anales.v77i4.12648

Castillo, S. J., Salinas, N., León, B. \& SÁnchez, I. (2006). Gentianaceae endémicas del Perú. En B. LeÓN, J. Roque, c. Ulloa, N. Pitman, P. M. Jorgensen \& A. CANO (eds.), El libro rojo de las plantas endémicas del Perú. Revista Peruana de Biología, Número especial 13: 339-354. https://doi.org/10.15381/rpb.v13i2.1860

Castillo, S. J. \& Pringle, J. S. (2018). Gentianella canoi S. J. Castillo \& J. S. Pringle sp. nov. (Gentianaceae), a new species from Perú. Anales del Jardín Botánico de Madrid 75: e068.

https://doi.org/10.3989/ajbm.2463 
Castillo, H., Albán, J. \& Castañeda, R. (2019). Importancia cultural de la flora silvestre de la provincia de Cajabamba, Cajamarca, Perú. Arnaldoa 26: 1047-1074.

Climate-Data.Org. (2019). Clima en el distrito de Jesús Cajamarca. https://es.climate-data.org/america-delsur/peru/cajamarca/jesus-875103/

Contreras, A. \& Méndez-Estrada, V. H. (2014). Fenología de la planta medicinal Valeriana prionophylla (Valerianaceae) en páramos de Costa Rica. Cuadernos de Investigación UNED 6: 223231. https://doi.org/10.22458/urj.v6i2.628

De Paula, B. M. F., Takeuchi, P. L. \& Vannucchi, H. (2018). Efecto del resveratrol, la catequina y el gingerol en la obesidad: evidencias incompletas. Nutrición Clínica en Medicina 12: 37-46.

EEBI-INIA (Estación Experimental, Baños del IncaInstituto Nacional de Innovación Agraria). (2018). Hoja de resultados de análisis de suelos. SU0550EEBI-18. Baños del Inca, Cajamarca.

Galán de Mera, A., Linares, E. \& Montoya, J. (2018). Mapa de vegetación de Cajamarca. Potencialidad de la vegetación para el uso de plantas medicinales. Universidad Privada Antonio Guillermo Urrelo, Municipalidad Provincial de Cajamarca, Asociación Los Andes de Cajamarca. Martínez Compañon eds. Cajamarca, Perú.

Galán de Mera, A., Linares-Perea, E., Martos, F., Montoya-Quino, J., Rodríguez-Zegarra, C. \& Torres-Marquina, I. (2019). Distribución bioclimática de plantas medicinales y sus principios activos en el departamento de Cajamarca (Perú). Boletín Latinoamericano y del Caribe de Plantas Medicinales y Aromáticas 18: 130-143.

Grupo Propuesta Ciudadana. (2014). Concesiones mineras en el Perú. Análisis y propuestas de política. Lima, Perú.

Huamán J. J., Torres, K. G., García, J, O., Lino, B. F., Méndez, E. A, Mariños, A. P., Quispe, C. A, Terrones, G. I., Morera, C. H., Livaque, J. A. \& Reyes, E. D. (2015). Efecto del consumo de Gentianella bicolor o "Corpus Huay" sobre la tolerancia oral a la glucosa y el perfil lipídico en adultos jóvenes. Revista Médica de Trujillo 11: $1-18$.

Isuasty-Torres, L., Pérez-Martínez, L. V. \& Vargas, O. (2014). Semillas y restauración ecológica. En VArgas, O. \& L.V. Pérez (eds.), Semillas de plantas de páramo: ecología y métodos de germinación aplicados a la restauración ecológica, pp. 43-61. Universidad Nacional de Colombia.
MagurRan, A. E. (2004). Measuring biological diversity. Blackwell Science Ltd. Oxford, United Kingdom.

Martínez-Arévalo, J. V. (2011). Domesticación de plantas medicinales. Agroproductividad 15: 3-7.

Mateucci, D. C. \& Colma, A. (1982). Metodologías para el estudio de la vegetación. Secretaría General de la OEA, Washington D.C.

MoRENo, C. (2001). Métodos para medir la biodiversidad. M\&T-Manuales y Tesis SEA, vol. 1. Zaragoza, España.

Mostacedo, B. \& Fredericksen, T. S. (2000). Manual de Métodos Básicos de Muestreo y Análisis en Ecología Vegetal. Proyecto de Manejo Forestal Sostenible (BOLFOR). Editora El País. Santa Cruz, Bolivia.

Nazar, J. \& Alva, E. (2015). Efecto del encalado en el crecimiento de Valeriana pilosa R. \& P. en Huanico, Cajamarca. Fiat Lux 11: 53-59.

Nibir, Y. M., Sumit, A, F., Akhand, A, A., Ahsan, N. \& Hossain, M. S. (2017). Comparative assessment of total polyphenols, antioxidant and antimicrobial activity of different tea varieties of Bangladesh. Asian Pacific Journal of Tropical Biomedicine 7: 352-357. https://doi.org/10.1016/j.apjtb.2017.01.005

OMS (Organización Mundial de la Salud). (2003). Directrices de la OMS sobre buenas prácticas agrícolas y de recolección (BPAR) de plantas medicinales. Ginebra, Suiza.

Pérez-Martínez, L. V., Rodríguez-Castillo, N. A., Vargas, O. \& Melgarejo, L. M. (2014a). Germinación y dormancia de semillas. En VARGaS, O. \& L. V. PÉrez-Martínez (eds.), Semillas de plantas de páramo: ecología y métodos de germinación aplicados a la restauración ecológica, pp. 63-113. Universidad Nacional de Colombia, Sede Bogotá.

Perez-Martínez, L. V., Rodríguez-Castillo, N. A., Melgarejo, L. M. \& Vargas, O. (2014b). Propagación por semilla de 13 especies de páramo. En O. Vargas \& L. V. Perez-Martínez (eds.), Semillas de plantas de páramo: ecología y métodos de germinación aplicados a la restauración ecológica, pp. 116-174. Universidad Nacional de Colombia, Sede Bogotá.

Pringle, J. S. (2008). Four New Species of Peruvian Gentianella (Gentianaceae). Novon: A J. for Botanical Nomenclature 18: 511-516. https://doi.org/10.3417/1997030

Pringle, J. S. (2017a). Four new species of Gentianella (Gentinaceae, Gentianeae, Swertiinae) from the Peru with a review of the taxonomy of the genus. Novon 25: 451-466. https://doi.org/10.3417/2016001 
Pringle, J. S. (2017b). Especies ginodioicas de Gentianella (Gentianaceae) en Colombia, Ecuador y Perú, con la descripción de G. quipuscoana, nueva especie del Perú. Arnaldoa 24: 9-18. https://doi.org/10.22497/arnaldoa.241.24101

Pulgar VidAL, J. (1996). Las ocho regiones naturales del Perú. PEISA, Lima, Perú.

Quiñones, M., Miguel, M. \& Aleixandre A. (2012). Los polifenoles, compuestos de origen natural con efectos saludables sobre el sistema cardiovascular. Nutrición Hospitalaria 27: 76-89.

Rao, N. K., Hanson, J., Dulloo, M. E., Ghosh, K., Novell, D. \& LaRinde, M. (2007). Manual para el manejo de semillas en bancos de germoplasma. Manuales para Bancos de Germoplasma $N^{\circ} 8$. Bioversity International, Roma, Italia.

RaYa-Pérez, J. C., Aguirre-Mancilla, C. L., Gil-Vega, K. \& Simpson, J. (2010). La domesticación de plantas en México: comparación de la forma cultivada y silvestre de Byrsonima crassifolia (Malpighiaceae). Polibotánica 30: 239-256.

Rodríguez, E. \& Rojas, R. (2006). El Herbario: Administración y Manejo de Colecciones Botánicas. 2 ed. Edit. por Vásquez, R. Missouri Botanical Garden, Perú.

SÁnChEZ, I. (2014). Plantas medicinales en los páramos de Cajamarca. En Cuesta, F., Sevink, J., Llambi, L. D., De Bièvre, B. \& J. Posner (eds.), Avances en investigación para la conservación de los páramos andinos, pp. 175-194. CONDESAN.
Seminario, A. \& Sánchez, I. (2010). Estado y factores de riesgo de la biodiversidad de especies vegetales medicinales en el Centro Poblado de Combayo, Cajamarca. Fiat Lux. 6: 23-34.

Seminario-Cunya, J., Rumay-Sánchez, L. D. \& Seminario-Cunya, A. (2016). Biología de Valeriana pilosa R. \& P. (Valerianaceae): una especie en peligro de extinción de las altas montañas de Perú. Boletín latinoamericano y del Caribe de plantas medicinales y aromáticas 15: 337-351.

The Plant List (2019). The plant list. A working list of all plant species. Royal Botanical Gardens. Kew Missouri Botanical Gardens. St Louis, Missouri, US. http://www.theplantlist.org/

Tropicos (2019). Tropicos.org. Missouri Botanical Garden. St Louis, Missouri, US. https://www. tropicos.org/Name/2734075

Vargas, O. \& Pérez-Martínez, L. V. (2014). Ecología de semillas en los páramos. En VARGas, O. \& L. V. PÉrez (eds.), Semillas de plantas de páramo: ecología y métodos de germinación aplicados a la restauración ecológica, pp. 21-42. Universidad Nacional de Colombia.

Vargas, M. G. \& SÁnchez, M. N. (2019). Diversidad de plantas herbáceas y subarbustivas en cerro Chucantí, provincia de Darién. Tecnociencia 21: 69-91.

Wolfe, K. L. \& LiU, R. H. (2003). Apple peels as a valueadded food ingredient. Journal of Agricultural and Food Chemistry 51: 1676-1683.

https://doi.org/10.1021/jf025916z 
